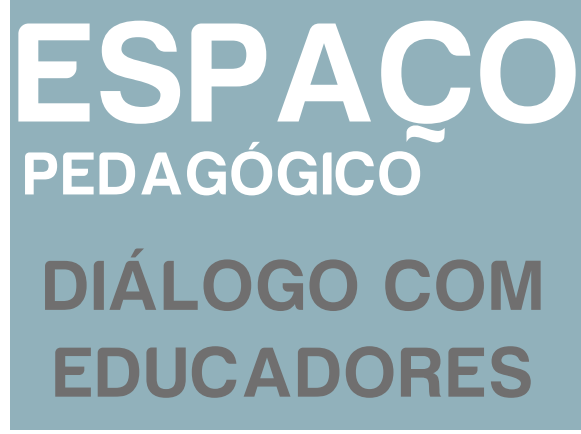




\section{Dialogue avec les éducateurs ${ }^{1}$}

Pierre Dardot

Christian Laval

Le premier numéro de la Revista Espaço Pedagógico du Programa de Pós-Graduação em Educação de la Universidade de Passo Fundo (PPGEDU/UPF) qui sera publié aux premiers quatre mois de 2021 a pour thème Éducation et socialisation. L'invitation à la collaboration de Pierre Dardot et Christian Laval dans la section Dialogue avec les éducateurs se justifie par la contribution de ces chercheurs à l'analyse sociologique des transformations en cours dans le monde, des multiples dimensions du néolibéralisme comme politique, mais aussi comme culture (modes de vie). Les contributions importantes de Dardot et Laval, notamment dans les ouvrages La nouvelle raison du monde : Essai sur la société néolibérale (2009), Commun : Essai sur la révolution au XXIe siècle (2014) et celui de Laval L'école n'est pas une entreprise : Le néo-libéralisme à l'assaut de l'enseignement public (2003), ont contribué aux études et réflexions dans des disciplines, séminaires, groupes de recherche, thèses et mémoires du PPGEDU. C'est un plaisir pour nous, les organisateurs, ainsi que pour la collaboration décisive du doctorant Regiano, de pouvoir établir ce dialogue plus direct à partir de questions articulées au thème de la revue et des recherches développées par Dardot et Laval.

Questions :

1. Le néolibéralisme produit un ensemble de transformations politiques et individuelles. Quelles sont-elles et comment ces transformations interfèrent-elles dans les processus de socialisation et d'individualisation?

Pendant longtemps, on a pensé que le néolibéralisme était avant tout une certaine forme de politique économique, qu'il consistait à revenir à un type de capitalisme très dur, à un régime de surexploitation des travailleurs. Ce n'est pas faux mais on passait à côté de l'originalité du néolibéralisme comme technologie de pouvoir pouvant s'appliquer à de multiples domaines, on manquait ainsi l'une

Recebido em: 15/09/2021 - Aprovado em: 15/09/2021.

http://dx.doi.org/10.5335/rep.v28i1.12804 
de ses caractéristiques principales : son ubiquité. Le néolibéralisme comme forme de pouvoir étend la logique capitaliste à la vie entière, et en particulier à la subjectivité, au domaine intime, à la représentation de soi. Ce n'est pas seulement un individualisme, une promotion de l'individu contre la société, car cela est trop général. Les individus sont mis dans des situations telles qu'ils doivent vivre dans un régime de compétition, et sont soumis à des pressions de toutes sortes afin qu'ils aillent toujours plus vite, qu'ils aillent au-delà d'eux-mêmes, comme le commande l'idéologie du sport de compétition. Pour obtenir ce résultat, il convient que la socialisation dans son sens le plus général d'intégration des valeurs collectives s'opère selon une logique concurrentielle plutôt que coopérative, et que l'individu fonctionne comme un capitaliste de lui-même, c'est-à-dire qu'il se valorise subjectivement comme s'il était un capital. En ce sens, l'éducation joue un rôle majeur dans la fabrication de ces nouvelles subjectivités. Cela a été l'enjeu de mes travaux personnels : je voulais convaincre dès les années 1990 le plus de gens dans les milieux éducatifs, et surtout les syndicalistes et les membres des mouvements pédagogiques que nous étions en train de vivre une grande mutation des systèmes scolaires, vers ce que j’ai appelé l'école néolibérale, et plus tard « la nouvelle école capitaliste ». Mais évidemment pour cela, il fallait montrer que le néolibéralisme, ce n'était pas seulement de l'économie, que c'était une norme générale et une forme d'existence qui allait de en plus trouver ses conditions dans la sphère éducative.

2. Des concepts tels qu'«entrepreneur de soi-même» ou «néo-sujet» composent le langage de l'éducation articulé aux discours méritocratiques. De quelle manière ces concepts indiquent-ils une dynamique qui cache des contradictions sociales, économiques et culturelles et qui tend à ne saluer que l'effort individuel?

Pierre Dardot et moi-même avons repris à Foucault ce concept si éclairant d' " entrepreneur de soi-même ", et à certains psychanalystes celui de « néo-sujet ". Nous n'avons donc que peu innové sur le plan terminologique et conceptuel. Ce qui nous semblait intéressant c'était de se dire qu'une certaine éducation, qu'une socialisation d'un certain genre, et qu'une vie aussi d'un certain style, pouvaient conduire à une nouvelle forme de subjectivité, ce que nous avons appelé la « subjectivité capitaliste ", liée à une identification à un capital personnel qu'il faut valoriser durant toute son existence, en recherchant à toujours accumuler «plus de valeur». Un peu comme si la logique du «toujours plus» si subtilement analysée par Marx dans Le Capital sortait du champ économique pour s'étendre à tout le 
fonctionnement social et à toutes les institutions. Cela va beaucoup plus loin que l'idéologie méritocratique propre aux systèmes scolaires de l'époque moderne, qui ont incorporé la morale du travail, de la peine, de la souffrance, pour obtenir une récompense. C'est toujours une normativité qui met en jeu une notion de "mérite", mais elle est celle de l'accumulation individuelle indéfinie et non plus celle de l'accomplissement d'une personnalité autonome, selon les valeurs de l'humanisme classique. On pourrait dire que l'individu idéal n'est plus l'homme de raison et de dignité que voulaient incarner autrefois certains bourgeois, membres des professions libérales éduquées, mais le sportif ou le spéculateur en bourse hyperconnecté qui a l'œil collé sur le tableau des performances de ses investissements physiques ou financiers. Ce mode de fonctionnement de l'institution scolaire ne profite qu'à ceux qui peuvent se permettre ce genre d'investissements dans la compétition. Il ne peut qu'accroître les inégalités entre les classes sociales devant l'école. A mes yeux, dans le champ éducatif, l'introduction d'une compétition exacerbée est à la fois l'effet sectoriel d'une logique normative générale et la réponse de l'institution à la demande d'égalité de la part des classes populaires et moyennes. La concurrence est une idéologie de combat et une technologie de pouvoir qui consolident la domination de ceux qui ont tous les atouts pour dominer et qui atomisent les dominés mis en concurrence entre eux pour obtenir les miettes du festin.

3. Les discours sur la compétitivité et l'efficacité sont incorporés dans les politiques éducatives de nombreux pays. Comment pouvons-nous avancer de manière critique pour déconstruire cette compréhension?

Comme vous le suggériez dans votre question précédente, au bout de la concurrence il y a toujours plus d'inégalité sociale. Mais il faudrait surtout essayer de convaincre les familles des classes moyennes qui adhèrent pour partie à un tel système concurrentiel, d'ailleurs de plus en plus coûteux pour elles, qu'elles sont perdantes à jouer un jeu qui détruit petit à petit le système public d'éducation en l'abandonnant aux plus pauvres. Car au bout du processus il y a la privatisation de l'éducation, donc l'endettement des familles et des étudiants, mais aussi l'appauvrissement des contenus culturels puisque seules les disciplines « rentables » sur le marché du travail seront enseignées. Mais ne nous trompons pas : le combat pour faire de l'éducation un véritable bien commun ne peut s'isoler d'une lutte beaucoup plus générale pour une société dont la valeur centrale ne serait plus l'accumulation mais ce que nous appelons le «commun ", entendant par là l'égali- 
té, la solidarité, la démocratie véritable, le libre accès à des ressources collectives indispensables, et en premier lieu à l'éducation. Et pour cela il vaut mieux payer des impôts que de payer des frais d'inscription de plus en plus élevés en vuie d'un rendement financier de plus en plus incertain.

4. La logique de la gestion d'entreprise capitaliste est en train d'être assumée dans de multiples institutions sociales et éducatives très fortement au Brésil d'aujourd'hui. Quels sont les risques de ce modèle plus largement pour une éducation républicaine et plus spécifiquement pour l'école?

Le risque, c'est évidemment la croissance de l'inégalité dans les conditions concrètes de d'éducation selon les établissements. L'école, à partir d'un certain degré d'inégalité, ne prépare abolument pas à une société favorisant un minimum d'intégration sociale, de communication même minimale entre les classes. Avec le néolibéralisme, nous vivons un grand recul de l'idée d'école commune, d'école pour tous, d'école inclusive. Les systèmes scolaires sous l'effet des logiques de marché sont en train de se fragmenter. Et la mobilité sociale, même si elle était faible, s'en trouve gravement atteinte. La sociologie de la reproduction a certes montré que l'éducation républicaine dont vous parlez n'avait pas remis en question le fonctionnement inégalitaire de l'école, mais elle montrait aussi que des progrès étaient possibles. Et le seul fait que dans les sociétés très scolarisées, la reproduction sociale passait par la médiation d'une sanction scolaire ouvrait une brèche dans les mécanismes quasi automatiques de cette reproduction quand elle s'opérait de façon excluivement familiale. Ainsi, dans beaucoup de pays, l'école était bien la seule chance, même si elle était réduite, de changer de position sociale. Et surtout, elle pouvait dans une certaine mesure donner des outils intellectuels aux enfants des classes populaires pour se défendre et conquérir de nouveaux droits.

Mais il existe encore un autre risque, qui est plus profond en un certain sens et qui touche à ce que le sociologue allemand Max Weber appelait «le type humain ». Faire entrer les nouvelles générations dans des entreprises éducatives, diffuser une culture d'entreprise dès le plus jeune âge comme le recommandent les grandes organisations économiques et financières dans le monde, c'est accélérer la transformation de l'humain en un être purement économique, un homo oeconomicus qui ne raisonne qu'en termes de coûts/bénéfices et qui a perdu tout sens des valeurs de solidarité, de culture, de dignité. Ce n'est donc pas seulement un mode de gestion des écoles plus efficace, c'est une culture qui se répand et qui modèle des 
subjectivités capitalistes. L'école devient alors non pas seulement une annexe du système économique dominant, mais une vraie matrice du capitalisme généralisé.

5. Comment avancer politiquement et pédagogiquement face à la tension entre l'individualisation et la socialisation intensifiées, dans le contexte actuel par le néolibéralisme «hyperautoritaire»?

Les enseignants doivent résister de toutes leurs forces à cette transformation et défendre les valeurs humaines les plus fondamentales, aujourd'hui dévaluées par l'esprit du capitalisme scolaire. En commençant peut-être par refuser de parler la langue du capitalisme scolaire, c'est-à-dire celle du management : " objectifs ", « culture du résultat ", " rendement ", " capital humain ", " employabilité ", « compétences", etc. S’en tenir au vocabulaire classique et normal de l'éducation humaniste, ce serait déjà beaucoup. Car comme disait Freud, si l'on cède sur le mot on cède sur la chose. On dira que c'est uniquement « défensif ». Oui, mais pour l'instant, les éducateurs sont agressés de toutes parts et doivent se défendre contre des formes subtiles d'invasion linquistique et conceptuelle. Elles sont subtiles parce que cette culture capitaliste a su utiliser beaucoup des apports des mouvements pédagogiques, en Europe et aux Etats-Unis, pour les détourner à son profit. S'il y a une attitude plus constructive à adopter, je crois qu'elle doit consister à réviser la tradition progressiste de l'éducation et à considérer d'un œil plus critique les formes idéologiques qui ont caractérisé certains courants ou certains auteurs regardés comme des fondements de la pédagogie progressiste. Je veux dire par là qu'il y a une hétérogénéité idéologique dans beaucoup de ces courants qui n'a pas été suffisamment analysée, ce qui a entraîné beaucoup de confusions dont a profité le capitalisme scolaire. Des éducateurs progressistes se sont fait les porteurs malgré eux de contenus individualistes, utilitaristes, psychologistes, au détriment de la sociologie critique et de l'esprit coopératif. Le travail à la fois théorique et pratique est immense : refonder une éducation démocratique au-delà des réutilisations néolibérales des pédagogies dites nouvelles. Et dans cette tâche considérable, la difficulté est qu'il faut tenir étroitement liées quatre dimensions : la création de situations éducatives réellement égales ; la construction d'une culture scolaire commune, socle solide à poser avant les spécialisations ultérieures ; la mise en place d'une pédagogie coopérative dès le plus jeune âge ; et une organisation démocratique des écoles dans lesquelles chacun, quelle que soit sa place d'éducateur, d'élève et de parent, puisse participer à une activité commuen. 
6. L'éducation a un rôle fondamental dans la formation du sujet démocratique. Pourquoi l'éducation ne réussit pas complètement à former par la démocratie, mais des sujets indifférents, autoritaires ou même «fascistes»?

D'abord l'école ne peut pas tout. C'est la société qui éduque d'abord. Si le fascisme est très présent dans une société, elle produit beaucoup de sujets fascistes, et c'est la même chose pour le racisme ou le machisme. Il faut arrêter de trop attendre de l'école. Ensuite, les systèmes scolaires quand ils se sont créé n'ont pas eu comme but de former des sujets démocratiques. Il s'agissait de former des chrétiens puis des citoyens obéissants de l'État nation. On oublie trop que l'idée d'émancipation des Lumières ne s'est pas réalisée concrètement dans les institutions réellement existantes. Ce sont l'Église et l'État qui ont fait l'École, et cette dernière en est restée profondément marquée, comme du reste toutes les institutions des sociétés européennes ou issues de la colonisation européenne. Et aujourd'hui c'est l'Entreprise qui veut modeler l'École. Donc, cette idée selon laquelle "L'éducation a un rôle fondamental dans la formation du sujet démocratique " a été très peu ou très mal incorporée dans les structures réelles de l'institution. Et elle est aujourd'hui chassée par l'idée selon laquelle il faut former des sujets économiques. Mais sans doute ce mythe progressiste de la formation du sujet démocratique est-il plus que jamais indispensable pour donner aux éducateurs et aux enseignants un sens à leur travail, mais encore faut-il qu'ils soient bien conscients que ce n'est pas la réalité. La question pratique, politiquement efficace, est donc plutôt de se demander comment ce mythe utile peut devenir réalité. Et donc comment faire pour que l'école ne produise plus ou produise moins de futurs sujets fascistes, racistes et machistes. La réponse ne peut tenir en quelques lignes, mais on peut dire de façon sans doute trop générale que ce qui est le plus précieux dans la tradition démocratique ce sont trois valeurs et façons d'être : 1) le sens de l'égalité réelle entre tous les jeunes, comme entre jeunes et adultes dans le respect des fonctions et des places différentes ; 2) le sens de la coopération démocratiquement organisée dans les apprentissages, les activités scolaires, la vie de la classe et de l'école ; 3) le sens de l'autonomie individuelle et collective, et surtout le sens du rapport qui existe entre l'autonomie individuelle et collective. Et tout ceci demande à s'incarner dans une organisation concrète, qui n'existe pas encore. L'éducation démocratique n'existe pas, elle est un projet. 
7. Quelles nouvelles perspectives le commun peut-il ouvrir, en tant que principe politique, pour penser des processus éducatifs émancipatoires? En ce sens, quel type d’imaginaire politique l'éducation peut-elle ou doit-elle encore aider à penser?

Dans l'ouvrage Commun nous n'avons pas développé la dimension éducative, et vous faites bien de poser la question. Je suis en train de mettre au point avec un ami syndicaliste, Francis Vergne, un nouveau livre, dont le titre provisoire est Éducation démocratique. Nous essayons de tracer les grandes lignes d'un nouveau système éducatif propre à une société effectivement démocratique, c'est-à-dire organisée selon le principe du commun. Nous entendons par «commun » non pas une caractéristique naturelle comme dans le droit romain, la théologie ou l'économie néoclassique mais comme un ordre juridico-politique qui a pour double modalité la participation démocratique à tous les niveaux et dans toutes les activités collectives, et la libre jouissance à égalité des ressources collectives jugées indispensables à l'épanouissement individuel et à la vie collective. L'un des aspects les plus importants, c'est le rapport que tous les élèves et les enseignants doivent avoir selon nous à la connaissance dans une société démocratique. Non pas comme un capital personnel qui assure une position de domination, mais un produit et une dimension de l'intelligence collective. Faire de la connaissance un bien commun, comprendre de façon sensible que la création de connaissance est affaire collective et que l'apprentissage a aussi une dimension collective, nous paraît essentiel. L'individualité ne peut se développer dans sa différence et sa singularité que dans l'échange et l'activité coopérative. En ce sens, le principe du commun peut servir à redonner de la cohérence aux pratiques pédagogiques et à la redistribution du pouvoir dans les établissements.

8. Comment considérez-vous la réception de l'œuvre Commun : Essai sur la révolution au XXIe siècle jusqu'à ce moment?

J'ai envie de vous répondre en plaisantant : le sous-titre du livre a anticipé une réception sur un siècle! Nous avons donc le temps d'en juger! Blague à part, la réception est assez curieuse. Le terme de "commun » est utilisé partout en France, en Europe et dans beaucoup de pays. Il y a un effet de mode considérable. Aux dernières élections municipales françaises, en mars 2020, beaucoup de listes ont adopté un nom sur le modèle de "Barcelone en commun ". C'est ainsi que j'ai pu voter pour une liste intitulée "Paris en commun ». Mais cela n'a que peu 
avoir avec notre proposition politique beaucoup plus radicale. Toute la question est de savoir si durant les décennies prochaines un nouveau corps doctrinal saura condenser trois types de lutte qui pour nous sont liées dans le revendication du commun : la lutte pour la démocratie, la lutte pour l'égalité sociale et la lutte pour sauver la planète. Le concept de commun pourrait contribuer efficacement à cette synthèse. Mais cela ne dépend pas des auteurs du livre mais des acteurs politiques et sociaux, s’ils en sentent la nécessité, si le lexique du commun et des communs leur paraît clarifier le sens qu'ils donnent à leurs combats.

Nota

1 Cette entrevue a été concédée par courriel aux organisateurs de l'édition 2021/1 de la revue Espaço Pedagógico, Angelo Vitório Cenci et Telmo Marcon. Il a été réalisé et traduit par le doctorant du Programa de Pós-Graduação em Educação de la Universidade de Passo Fundo (PPGEDU/UPF) qui participe au Groupe d'études sur le néolibéralisme et les alternatives (GENA) coordonné par les auteurs avec lesquels nous avons dialogué. 